\title{
Is deployement of diagnostic test alone enough? Comprehensive package of interventions to strengthen TB laboratory network: three years of experience in Burkina Faso
}

Riccardo Alagna ${ }^{1 *}$ D, Adjima Combary², Elisa Tagliani ${ }^{2}$ Léon Tinnoga Sawadogo ${ }^{2}$, Tandaogo Saouadogo ${ }^{2}$, Souba Diandé ${ }^{2}$, Francis Ouedraogo ${ }^{2}$ and Daniela Maria Cirillo ${ }^{1}$

\begin{abstract}
Backgrounds: The laboratory plays a critical role in tuberculosis (TB) control by providing testing for diagnosis, treatment monitoring, and surveillance at each level of the health care system. Weak accessibility to TB diagnosric services still represents a big concern in many limited resources' countries. Here we report the experience of Burkina Faso in implementing a comprehensive intervention packages to strengthen TB laboratory capacity and diagnostic accessibility.

Methods: The intervention lasted from October 2016 to December 2018 and focused on two main areas: i) development of strategic documents and policies; ii) implementation of TB diagnostic technology.

National TB laboratory data were collected between 2016 and 2018 and evaluated according to five programmatic TB laboratory indicators: i) Percentage of notified new and relapse TB cases with bacteriological confirmation; ii) Percentage of notified new and relapse TB cases tested by Xpert MTB/RIF; iii) Percentage of notified, bacteriologically confirmed TB cases with a drug susceptibility testing (DST) result for rifampin; iv) Percentage of notified MDR-TB cases on the estimated number of MDR-TB cases; v) The ration between the number of smear microscopy and Xpert MTB/RIF tests. We compared these indicators between a 1 year (2016-2017) and 2 years (2016-2018) timeframe.
\end{abstract}

Results: From 2016 to 2018, the percentage of bacteriologically confirmed cases increased from 67 to $71 \%$. The percentage of new and relapse TB cases notified tested by Xpert MTB/RIF increased from 18\% in 2016 to $46 \%$ in 2018 and the percentage of bacteriologically confirmed cases with an available DST result for rifampicin increased from 27\% in 2016 to $66 \%$ in 2018.. The percentage of notified MDR-TB cases on the estimated number of MDR-TB cases in 2018 increased from 43\% in 2016 to 78\% in 2018. In 2018, the ratio between the number of smear microscopy and Xpert MTB/RIF tests decreased from 53\% in 2016 to 21\% in 2018.

(Continued on next page)

\footnotetext{
* Correspondence: alagna.riccardo@hsr.it

${ }^{1}$ IRCCS San Raffaele Scientific Institute, Milan, Italy

Full list of author information is available at the end of the article
} 
(Continued from previous page)

Conclusion: We demonstrated that the implementation of a comprehensive package of laboratory strengthening interventions led to a significant improvement of all indicators. External technical assistance played a key role in speeding up the TB laboratory system improvement process.

Keywords: Tuberculosis, International cooperation, Technical assistance, Diagnostic

\section{Background}

The laboratory plays a critical role in tuberculosis (TB) control by providing testing for diagnosis, treatment monitoring, and surveillance at each level of the health care system. Despite representing an integral part of a continuum of care by providing evidence for decision making in clinical practice, diagnostic servicses are the weakest link in the cascade of care influencing only 60$70 \%$ of the healthcare decisions [1]. The diagnostic gap is a common thread of many infectious diseases, but it is much higher for TB [2].

Globally in 2019, there was a gap of about 2.9 millions between the number of incident and notified TB cases in the same year [3]. The missed cases were either not diagnosed or diagnosed but not reported. Despite the development of diagnostic tests able to provide rapid and accurate detection of TB and drug-resistant TB, poor accessibility to the health care system is still a big concern in many settings $[4,5]$. In addition, on-field implementation studies have shown that diagnostic tests alone may fail to meet the expected impact on the TB care cascade if not implemented within the context of a strengthened health system [6].

Development of new TB diagnostic, treatment, and prevention tools has increased substantially over the last decades, leading to more than 20 new or updated World Health Organization (WHO) guidelines on different aspects of TB care [3, 7-9].

As a result of these rapid changes, National TB Programmes (NTP) have experienced difficulties in the prompt adoption and full implementation of TB laboratory policies and strategies $[6,10]$. In this context, the WHO plays a crucial role in directing the international public health efforts through the coordination of technical assistance programmes to countries, partnerships, and initiativesaimed at facilitating the translation of policies into practice. The TB Supranational Reference Laboratory Network (SRLN) is a key WHO technical resource in supporting the strengthening of laboratory capacity in high TB burden countries [11].

The Emerging Bacterial Pathogens Unit (EBPU) of the Fondazione Centro San Raffaele part of San Raffaele Scientific Institute of Milan, Italy, was appointed as SRL and as WHO Collaborating Centre for TB laboratory strengthening (ITA-98) in 2006 and 2013, respectively. From 2016 to 2018, the EBPU-SRL Milan, thanks to the
United States Agency for International Development (USAID) financial support, has served as a technical partner to the WHO Global TB Program to strengthen the TB diagnostic capacities and to support the DrugResistance Surveillance (DRS) activities in selected countries, including Burkina Faso..

The objective of the present study is to determine the impact of implementing a comprehensive package of interventionson the TB laboratory capacity and accessibility in Burkina Faso.

\section{Methods \\ Setting}

In 2016, the Burkina Faso TB three-tier laboratory network consisted of a National TB reference laboratory (NRL), 13 regional laboratories, and 97 peripheral laboratories. Both fluorescence and conventional light microscopy were the main TB diagnostic methods used. The Xpert MTB/RIF assay (Cepheid, Sunnyvale CA) was first introduced in the country in June 2013 with the placement of one 4-module GeneXpert machine (GX4) at the TB NRL. Subsequently, in October 2016, two additional GX4 instruments were installed at two regional laboratories. By November 2016, the GeneXpert network was expanded with additional 12 GX4 machines resulting in a total of 15 instruments covering all regions in the country.

Routine TB examination consisted on clinical evaluation, sputum smear microscopy, and chest radiography. Patients at high-risk of multidrug-resistant TB (MDRTB), children, and people living with HIV (PLHIV) were tested by Xpert MTB/RIF, and patients with rifampin resistance results were referred to the NRL for genotypic testing for first-line anti-TB drugs.

\section{Intervention strategy}

From October 2016 to December 2018, the EBPU-SRL Milan has provided extended and highly specialized technical assistance (TA) to the NTP and the national TB laboratory network of Burkina Faso. The TA focused on two main areas: i) development of strategic documents and policies; ii) implementation of TB diagnostic technologies (Fig. 1).

i) Development of strategic documents and policies

The National TB Laboratory network assessment carried ot in 2017 led to the identification of the 


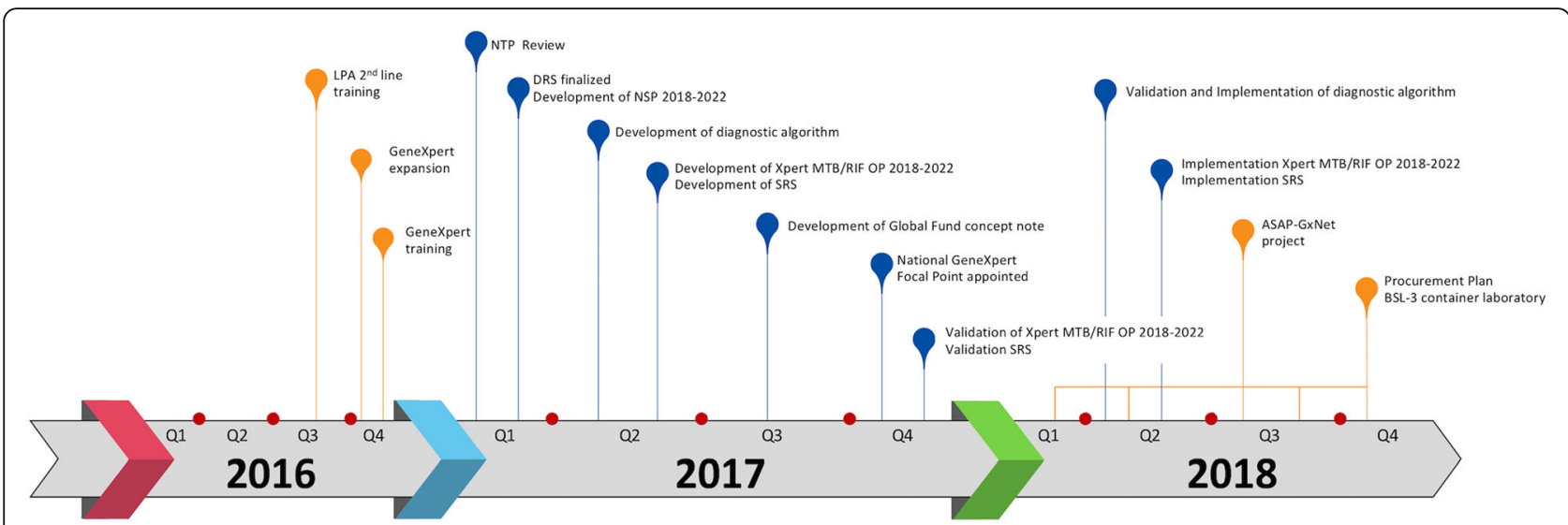

Fig. 1 Timeline of TA activities carried out between October 2016 to December 2018. Orange dots represent the implmenetation of TB diagnostic guidelines and technology; Blue dots represent the development of strategic documents and policies. Line Probe Assay (LPA); National TB Programme (NTP); Drug-resistance Survey (DRS); Operational Plan (OP); Sample Referral Strategy (SRS): Biosafety Level-3 (BSL-3)

strengths and weaknesses of the overall system, while the DRS study provided information on national anti-TB drug resistance prevalence. Altogether, this information guided the NTP to determine the priority actions to be implemented and constituted the foundations for developing new or revising existing national policies and strategic plans such as the five-years National Strategic Plan (NSP) 2018-2022. Diagnostic algorithms had also been reviewed to move toward the END TB Strategy goal of universal access to rapid diagnostics and DST (Supplementary material: Figure S1, S2.1-S2.4). To this purpose, the NTP developed an Xpert MTB/ RIF operational plan for the years 2018-2022 to strengthen the national GeneXpert network. Special attention had also been paid to the sample referral and transport system to enable the timely diagnosis of patients living in the countries remote areas. This comprehensive package of strategic and operational plans facilitated the development of the concept note for the Global Fund grant cycle 20182020 to ensure the proper financing of the TB laboratory priorities.

ii) Implementation of TB diagnostic technologies

The development of strategic documents was interconnected with activities aimed at strengthening the TB national diagnostic services. By November 2016, the GeneXpert network was expanded with additional twelve 4-modules machines resulting in a total of 15 instruments covering all regions. A total of 60 laboratory technicians were trained to operate the GeneXpert machines, and more than 200 clinicians were instructed on TB patients referral and initiation of appropriate treatment based on Xpert results. To ensure the effective introduction of Xpert MTB/RIF test within the diagnostic routine, a pilot operational research project financed by the STOP TB Partnership named "Applying a Standardized Approach to Strengthen Performances of GeneXpert Networks" (ASAP-GxNet) was started [12]. The project aimed at strengthening the local managerial skills and at assessing in a standardised way the functionality of the GeneXpert network. Finally, the expansion of the GeneXpert network was accompanied by the strengthening of the diagnostic capacity at the NRL [13]. The activities included the implementation of Line Probe Assay (LPA) for second line drugs and the development of a procurement plan for a Biosafety Level-3 (BSL-3) container laboratory to be used for liquid culture and Drug-Susceptibility Testing (DST).

\section{Data sources and analysis}

The NTP provided the TB case notification data from the national TB electronic database from 2016 to 2018. All data were anonymized and no sensitive patient information were used for data analysis. Data included TB notifications among new and previously treated patients stratified by type of diagnosis (i.e. clinical or bacteriological), test type (smear microscopy, Xpert MTB/RIF) and result. Access to data has been granted to the TB Surpanational Reference Laboratory of Milan by the National TB Programme of Burkina Faso within the framework of collaboration agreement signed between the two parties in 2011.

Quantitative data per year were imported to an Excel spreadsheet and analysed according to five programmatic TB laboratory indicators: i) Percentage of notified new and relapse TB cases with bacteriological confirmation; ii) Percentage of notified new and relapse TB cases tested with Xpert MTB/RIF assay; iii) Percentage of 
notified, bacteriologically confirmed TB cases with DST results for rifampin; iv) Percentage of notified MDR-TB cases on the estimated number of MDR-TB cases; v) Ratio between the number of smear microscopy and Xpert MTB/RIF tests.

TB notification data was used to calculate the five indicators and trends were compared over a 3 years timeframe.

\section{Results}

To assess the impact of the development and implementation of TB diagnostic guidelines and the effectiveness of the TA provided by the SRL Milan, we monitored the following five indicators over a three-years timeframe, from 2016 to $20 / 20$ :

i) Notified new and relapse TB cases with bacteriological confirmation in 2016, 2017 and 2018

A total of 5918 and 5839 new and relapse TB cases were notified in 2016 and 2017, respectively. Of them, 3993 (67\%) and 4044 (69\%) were bacteriologically confirmed through smear microscopy and/or Xpert MTB/ RIF (Fig. 2). In 2018, out of the 6166 new and relapse TB cases notified, 4288 (71\%) were bacteriologically confirmed through smear microscopy and/or Xpert MTB/ RIF (Fig. 2).

ii) Notified new and relapse TB cases tested with Xpert MTB/RIF in 2016, 2017 and 2018

The number of new and relapse TB cases notified tested with Xpert MTB/RIF remained stable in 2016 and
2017 with a totale of $1094 / 5918$ (18\%) and 1147/5839 (20\%) in 2016 and 2017 respectively. Only in 2018, we observed a much higher increase in the number of notified TB cases that received an Xpert MTB/RIF test, up to $2826 / 6166$ (46\%) (Fig. 2).

iii) Notified, bacteriologically confirmed TB cases with DST result for rifampicin in 2016, 2017 and 2018

The number of bacteriologically confirmed TB cases with an available DST result for rifampicin increased from $27 \%$ in $2016(1094 / 3993)$ to $28 \%$ in 2017 (1147/ 4044) (Fig. 3). Notably, the number of bacteriologically confirmed cases with an available DST result for rifampicin increased up to 66\% (2826/4288) in 2018 (Fig. 3).

iv) Notified MDR-TB cases in 2016, 2017 and 2018

The number of notified MDR-TB cases (confirmed by Genotype MTBDRplus VER 2.0) over the number of estimated MDR-TB cases increased from 43\% (58/136) in 2016 to $47 \%(63 / 134)$ in 2017. In 2018, we observed a much higher increase in the ratio between notified and estimated MDR-TB cases, upt to 78\% (102/131).

xxiiRatio between the number of smear microscopy and Xpert MTB/RIF tests

The utilization of Xpert MTB/RIF test in routine practice has been assessed by calculating the ratio between the number of smear-microscopy and Xpert MTB/RIF tests performed annually. This ratio decreased from $53 \%$ $(58,510 / 1105)$ to $51 \%(61,755 / 1200)$ in 2016 and 2017

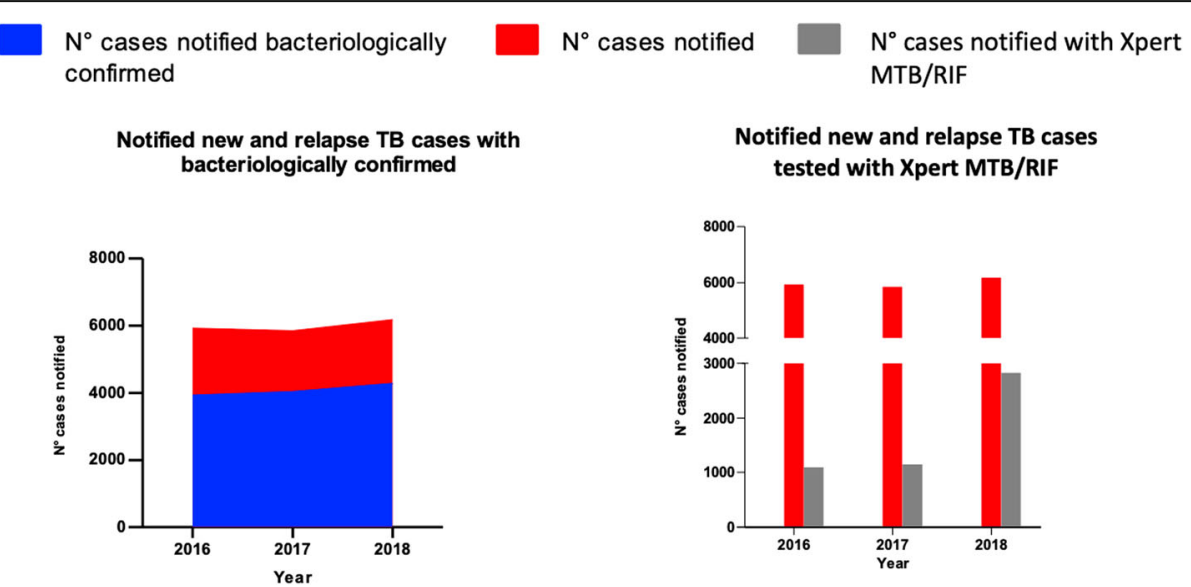

Fig. 2 Laboratory indicators I and II. On the left, trend of number of new and relapse TB cases notified between 2016 and 2018 stratified based on type of diagnosis. On the right, the absolute number and percentage of new and relapse TB cases notified from 2016 to 2018 tested with Xpert MTB/RIF; in red notified cases clinically diagnosed, in blu notified cases Xpert MTB/RIF diagnosed 
Notified, bacteriologically confirmed TB cases with DST results for rifampicin

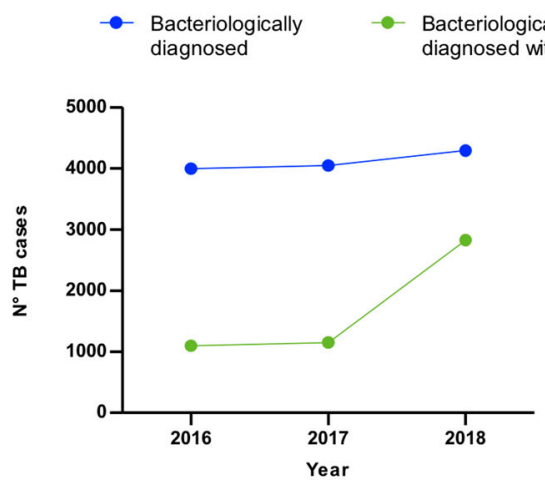

MDR-TB cases

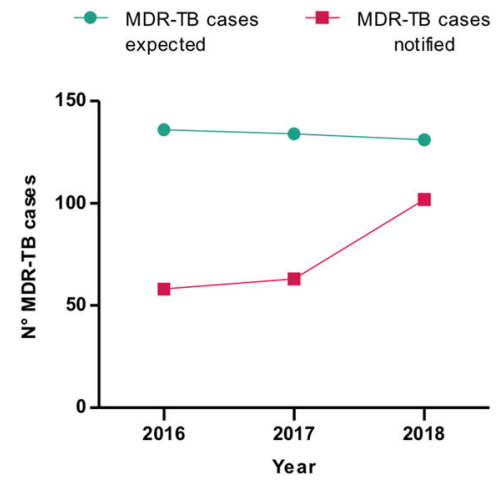

Fig. 3 Laboratory indicators III and IV. On the left, number of notified bacteriologically confirmed TB cases compared to the number of bacteriologically confirmed TB cases with rifamicin result. On the right, number of MDR-TB cases notified over the number of estimatd MDR-TB cases

respectively. In 2018, the ratio between the number of smear microscopy and Xpert MTB/RIF test substantially decreased down to $21 \%(59,714 / 2826)$.

\section{Discussion}

In this paper, we demonstrated that broader access to diagnostic services requires a comprehensive package of interventions that address multiple needs. This evidence is clearly supported by the improvement of all five indicators we monitored over the three-years timeframe. By the end of 2016, Burkina Faso experienced a 5-time increase in the number of 4-module GeneXpert instruments. This expansion was crucial to decentralize the diagnostic capacity of the country, but still not sufficient to drastically improve the evaluated indicators over a 1 year timeframe (2016-2017). We instead observed a strong improvement of the five indicators by the end of 2018, after the implementation of laboratory guidelines and strategies. Nonetheless, while all of evaluated indicators showed a substantial increase over time, the overall number of new and relapse TB cases that were bacteriologically confirmed showed a smaller change over the two-years timeframe (2016 to 2018). This can be explained by the fact that access to Xpert MTB/RIF testing was initially limited to smear positive patients at highrisk of MDR-TB, to avoid the rapid overload of the network and to promote a more gradual move toward universal DST.

Also the provision of technical assistance played an important role in speeding up the process of increasing diagnostic accessibility. This is shown by the change in the ratio between the number of smear microscopy and Xpert MTB/RIF tests performed. This indicator can be used to monitor how a country moves with regard to Xpert usage when compared to other settings [10, 14]. Between 2017 and 2018, Burkina Faso substantially increased the utilization of GeneXpert over microscopy, which resulted in a 30\% decrease of the smear/Xpert ratio. Notably, this process was much faster as compared to other African countries with more experience on Xpert implementation such as Mozambique, Uganda, DR Congo, and Zimbabwe [10].

It is undoubtedly that interventions had a positive effect on the evaluated indicators and external assistance has played a fundamental role in speeding up the TB laboratory system improvement process. However, we acknowledge that additional programmatic factors, other than those evaluated in this work, such as community engagement to promote health seeking behaviours, improved TB communication strategies and support to TB advocacy groups, could have contributed to the improvement of the TB indicators.

\section{Conclusion}

The experience of Burkina Faso shows that the countrywise implementation of laboratory guidelines is a requirement to rapidly advance the provision of TB laboratory services. We believe that, the lessons learnt over this three-years timeframe will be useful to other NTPs that aim to achieve concrete goals in strengthening their national TB laboratory networks. One of the key lessons learnt is the need for country commitment. These results could have not been achieved without a strong national commitment as this represents a sine qua non condition to ensure that the planned activities have a continuum on national TB control strategy. Another key lesson learnt is related to the need of building a roadmap of priority interventions guided by a comprehensive analysis that include the country's epidemic 
situation, as well as the assessment of financial and, human resources and available network infrastructure. In ou specific context, timing played a crucial role, as Burkina Faso was able to achieve most of its results by conducting this comprehensive analysis and development of key operational plans the year before the Global Fund grant development. This was critical to secure the necessary funding to implement priority activities. Together with this clear activity plan, technical assistance played a key role in boosting the achievement of the obtained outcomes. Importantly, TA needs to be planned in advance with the country and must be provided over an adequate period of time rather than being delivered ondemand or through a single interaction. Supporting countries with a comprehensive package of technical assistance interventions that cover the entire donor's grant cycle is preferable over a fragmented TA since this helps ensuring continuity while at the same time increasing the pace of change. Estimating TA financial needs is complex. Technical partners must work togehter with the NTP in identifying upfront the country needs for the implementation of the priority activities during the donor's grant cycle. In this context, TA acts as an intermediary to best align and leverage donor's and recipient's needs. Security of funds for external technical assistance has multiple benefits as it allows to work with the same TA provider over a prolonged period of time and, therefore, to build a strong relationship between the partners and facilitate the systematic transfer of skills. Besides the TA plan and secured funding, also the consultant individual capacity and skills in assisting the country is a key factor, as it should promote the establishment of an environment fostering the autonomous development of the country staff rather than a dependency culture.

\section{Recommendations}

The experience from Burkina Faso can help outlining some recommendations which may inform countries with similar challenges on future technical assistance interventions to strengthen laboratory system:

- Conduct a comprehensive laboratory network assessment to identify TB laboratory needs;

- Identify a package of actions that are feasible and acceptable to both those receiving and those delivering supports;

- Ensure that TB laboratory needs are translated into the National Strategic Plan;

- Update the national diagnostic algorithms to ensure optimal utilization of laboratory resources;

- Develop a national sample referral system to improve accessibility of laboratory services;
- Develop an Xpert Operational Plan based ASAPGxNet assessment tools;

- Ensure that policies are effectively implemented by health professional capacity building;

- Ensure country ownership by enhancing country's capacity to determine its own development and decide its priority activities. Ownership also leads to high-level intention to maintain and achieve results.

- Ensure engagement of an external consultant that fits with the demands of health development in lowresource settings and has the ability to enhance an autonomous development environment rather than a dependency culture;

- Plan external technical assistance upfront and ensure its provision over a prolonged period rather than a request that is answered immediately or through a single interaction;

- Ensure robust funding mechanism to support external technical assistance providers as this allows external providers to appropriately shape the activities according to the country needs and to attend the expected benefit.

\section{Abbreviations}

BSL-3: Biosafety Level-3; DRS: Drug-Resistance Surveillance; DST: Drugsusceptibility testing; EBPU: Emerging Bacterial Pathogens Unit; GX4: GeneXpert machine 4-module; LPA: Line Probe Assay; MTB: M. tuberculosis; MDR-TB: Multidrug-resistant TB; NRL: National TB reference laboratory; NSP: National Strategic Plan; NTP: National TB Program; OP: Operational Plan; PLHIV: People living with HIV; RIF: Rifampicin; SRLN: TB Supranational Reference Laboratory Network; SRS: Sample Referral Strategy; TA: Technical assistance; TB: Tuberculosis; USAID: United States Agency for International Development; WHO: World Health Organization

\section{Supplementary Information}

The online version contains supplementary material available at https://doi. org/10.1186/s12879-021-06012-y.

\section{Additional file 1.}

\section{Acknowledgements}

The authors are grateful to the National Tuberculosis Programme Team of Burkina Faso and Dr. Paolo Miotto from IRCSS San Raffaele Scientific Institute.

\section{Authors' contributions}

RA led the design of the study, advised on data analysis and interpretation and drafted the manuscript. AC participated in the design of the study, advised on data analysis and interpretation, and drafted manuscript. LTS, ST, $\mathrm{SD}$, FO contributed to data analysisand interpretation, and reviewed the manuscript. ET participated in the interpretation of the data and in writing the manuscript. DMC advised on data analysis, participated in data interpretation and writing of the manuscript. All authors read and approved the final manuscript.

Funding

Current research has not received any funding. Technical assistance activities have been financed by WHO through financial support of USAID.

Availability of data and materials

The datasets generated and/or analysed during the current study are not publicly available due to data protection requirements but are available from the corresponding author on reasonable request. 


\section{Declarations}

\section{Ethics approval and consent to participate}

Ethics approval was deemed as not necessary from the Comité national d'éthique pour la recherche en santé (CNRES), the Direction de la protection de la santé de la population (DPSP) of Ministry of Health of Burkina Faso and the Comitato Etico of I.R.C.C.S Ospedale San Raffaele as the intervention, approved by the DPSP, was conducted as part of the standard of care provided by the National TB Programme of Burkina Faso. In addition, all the collected data were anonymized and no patients sensitive information were used in data analysis. Access to data has been granted to the TB

Surpanational Reference Laboratory of Milan by the National TB Programme of Burkina Faso within the framework of collaboration agreement signed between the two parties in 2011.

\section{Consent for publication}

Not applicable.

\section{Competing interests}

The authors declare that they have no competing interests.

\section{Author details}

${ }^{1}$ IRCCS San Raffaele Scientific Institute, Milan, Italy. ${ }^{2}$ National Tuberculosis

Program, Ouagadougou, Burkina Faso.

Received: 4 February 2020 Accepted: 23 March 2021

Published online: 13 April 2021

\section{References}

1. Regan M, Forsman R. The impact of the laboratory on disease management Dis Manag. 2006;9(2):122-30. https://doi.org/10.1089/dis.2006.9.122.

2. Subbaraman R, Nathavitharana RR, Mayer KH, Satyanarayana S, Chadha VK, Arinaminpathy $\mathrm{N}$, et al. Constructing care cascades for active tuberculosis: a strategy for program monitoring and identifying gaps in quality of care. PLoS Med. 2019;16(2):e1002754. https://doi.org/10.1371/journal.pmed.1002 754.

3. WHO. Global Tuberculosis Report 2020. 2020. https://doi.org/10.3917/spub. 092.0139. Publication date: 15 October 2020.

4. Zumla A, Abubakar I. Improving access to multi-drug resistant tuberculosis diagnostic and health services for refugees and migrants. BMC Med. 2018; 16(1):221. https://doi.org/10.1186/s12916-018-1218-0.

5. Lin $\mathrm{H}-\mathrm{H}$, Dowdy D, Dye C, Murray M, Cohen T. The impact of new tuberculosis diagnostics on transmission: why context matters. Bull World Health Organ. 2012;90(10):739-47. https://doi.org/10.2471/BLT.11.101436.

6. Albert H, Nathavitharana RR, Isaacs C, Pai M, Denkinger CM, Boehme CC Development, roll-out and impact of Xpert MTB/RIF for tuberculosis: what lessons have we learnt and how can we do better? Eur Respir J. 2016;48(2): 516-25. https://doi.org/10.1183/13993003.00543-2016. Epub 2016 Jul 13.

7. Nasser SM, Cooke G, Kranzer K, Norris SL, Olliaro P, Ford N. Strength of recommendations in $\mathrm{WHO}$ guidelines using GRADE was associated with uptake in national policy. J Clin Epidemiol. 2015;68(6):703-7. https://doi. org/10.1016/j.jclinepi.2014.11.006. Epub 2014 Dec 13.

8. Brigden G, Nhung NV, Skrahina A, Ndjeka N, Falzon D, Zignol M. Advances in clinical trial design for development of new TB treatments-translating international tuberculosis treatment guidelines into national strategic plans: experiences from Belarus, South Africa, and Vietnam. PLoS Med. 2019;16(10): e1002896. https://doi.org/10.1371/journal.pmed.1002896.

9. Lienhardt C, Nahid P. Advances in clinical trial design for development of new tb treatments: a call for innovation. PLoS Med. 2019;16(3):e1002769. https://doi.org/10.1371/journal.pmed.1002769.

10. Cazabon D, Pande T, Kik S, Van Gemert W, Sohn H, Denkinger C, Qin ZZ, Waning B, Pai M. Market penetration of Xpert MTB/RIF in high tuberculosis burden countries: A trend analysis from 2014-2016. Gates Open Res. 2018;2: 35. https://doi.org/10.12688/gatesopenres.12842.2.

11. Gilpin C, Mirzayev F. Tuberculosis supranational reference laboratories: a global approach. Clin Chest Med. 2019;40(4):755-62. https://doi.org/10.1016/ j.ccm.2019.07.005.

12. Alagna R, Sawadogo TL, Combary A, Diandé S, Cirillo DM. ASAP-GxNet project in Burkina Faso: fulfil country capacity gaps to ensure efficient utilisation of GeneXpert instruments in tuberculosis care and cascade. ERJ
Open Res. 2019;5(1):00150-2018. https://doi.org/10.1183/23120541.00150-2 018.

13. Alagna R, Sawadogo TL, Tagliani E, Combary A, Cirillo DM. Applying a Standardized Approach to Strengthen Performances of GeneXpert Networks programme: lessons learned from Burkina Faso, 2019. ERJ Open Res. 2020; 6(4):00283-2020. https://doi.org/10.1183/23120541.00283-2020.

14. Cazabon D, Suresh A, Oghor C, Qin ZZ, Kik SV, Denkinger CM, Pai M. Implementation of Xpert MTB/RIF in 22 high tuberculosis burden countries: are we making progress? Eur Respir J. 2017;50(2):1700918. https://doi.org/1 0.1183/13993003.00918-2017.

\section{Publisher's Note}

Springer Nature remains neutral with regard to jurisdictional claims in published maps and institutional affiliations.
Ready to submit your research? Choose BMC and benefit from:

- fast, convenient online submission

- thorough peer review by experienced researchers in your field

- rapid publication on acceptance

- support for research data, including large and complex data types

- gold Open Access which fosters wider collaboration and increased citations

- maximum visibility for your research: over $100 \mathrm{M}$ website views per year

At BMC, research is always in progress.

Learn more biomedcentral.com/submissions 